prime or composite character and, if composite, its complete resolution into prime factors. The emphasis is here essentially on the adjective "immediate", for tables (by D. N. Lehmer) are available giving the smallest prime factor of every integer, less than $10,017,000$, which is not divisible by $2,3,5$, and so allowing the factorization of any integer up to this limit. But the labour of producing the factors may still be very great, so much so indeed that the British Association Tables Committee issued in 1935 a table of complete decompositions of all integers less than 100,000 , which was then the longest list of decompositions of consecutive integers. Kaván's work is more than two and a half times as extensive.

The tables are arranged to read horizontally from left to right across the page. The leading figures are found in the left-hand column, the terminal digit $0,1, \ldots, 9$ being across the top of the page. This was the arrangement suggested by Cayley, and seems easier of reference than the Chinese method of vertical columns read downwards which has also been used in this type of table. In such tables the tabular value is only another aspect of the argument; we have in fact a table of $x$ with $x$ as argument. The printing is clear, and a bold clarendon type distinguishes the prime numbers; a captious critic might suggest that figures with heads and tails would have been even more appropriate for the tabular values.

The method of calculation is described in Dr. Beer's preface, and, what is more important from the user's point of view, the precautions which have been taken to ensure the greatest possible accuracy. All the decompositions were checked by re-multiplication. The decompositions were compared with those in the tables of Inghirami (1832), of Goldberg (1862), and of Chernac (1811). After printing, a last check was made by comparing the decompositions of all numbers $x$ with those of $2 x$, $3 x, 5 x$. Those numbers which evaded this check were again compared with Chernac's values.

The table was used by Kaván as a basis to calculate the function $\varphi(n)$ mentioned above, in the course of which errors in the table would have been revealed. A list of 24 errors is given at the end of the book, together with a version of certain entries where the type impression is poor. It is therefore reasonable to suppose that the work will contain few, if any, undetected errors.

All those responsible may be congratulated on a fine piece of work, nobly conceived, ably executed and worthily printed.

\section{M. Milne-Thomson.}

\title{
Developments in Theories of Integration
}

Modern Theories of Integration

By H. Kestelman. Pp. viii +252 . (Oxford : Clarendon Press; London: Oxford University Press, 1937.) $17 s .6 d$. net.

THE author of this book complains that while Lebesgue integration is everywhere firmly established as an essential part of the theory of functions, its treatment in English text-books "remains unsatisfactory and uninfluenced by the simplified treatment of Carathéodory". The account of the Lebesgue integral he gives follows closely the lines of Carathéodory's "Vorlesungen über reelle Funktionen". The Lebesgue theory of measure forms the basis of a theory of integration from which the principal defects of the Riemann theory are absent. The Lebesgue integral is a generalization of the Riemann integral, and the Lebesgue theory of measure allows an enormous extension of the solution of the problem of areas only partially solved by the Riemann integral (or by the theory of Jordan content).

The author devotes a chapter to Riemann integration in order both to establish conditions in which it exists and to discuss its relation to problems such as the integration of derivatives and of limit functions in general. It is only by appreciating the circumstances in which the Cauchy-Riemann integral is insufficient that Lebesgue's achievement can be appreciated.

The extensions of the Lebesgue integral are traced from Hölder's definition to that of Denjoy ; and the general Denjoy integral is developed without the use of transfinite numbers. This treatment is derived by the author from a paper by P. Romanovski. A chapter on Fourier series illustrates the application of Lebesgue theory to a branch of analysis for which it has been of the utmost importance.

The book begins with a chapter on the definitions and elementary theorems of the theory of aggregates, particularly of sets of points, and develops them so far as is necessary for an understanding of the theory of the Lebesgue integral. Here, proofs of several theorems depending on the existence of certain aggregates for which no rule of 'construction' was forthcoming, are legitimatized by the introduction of Fraenkel's axiom of selection. This axiom is specially invoked to prove that every infinite set contains a sequence of distinct points and it is often used implicitly in existence theorems.

A. v. Z. 